\title{
On the origin of the anomalous ultraslow solvation dynamics in heterogeneous environments
}

\author{
KANKAN BHATTACHARYYA ${ }^{1, *}$ and BIMAN BAGCHI ${ }^{2, *}$ \\ ${ }^{1}$ Department of Physical Chemistry, Indian Association for the Cultivation of Science, Jadavpur, \\ Kolkata 700032 \\ ${ }^{2}$ Solid State and Structural Chemistry Unit, Indian Institute of Science, Bangalore 560012 \\ e-mail: pckb@iacs.res.in; bbagchi@sscu.iisc.ernet.in
}

\begin{abstract}
Many recent experimental studies have reported a surprising ultraslow component (even $>10 \mathrm{~ns}$ ) in the solvation dynamics of a polar probe in an organized assembly, the origin of which is not understood at present. Here we propose two molecular mechanisms in explanation. The first one involves the motion of the 'buried water' molecules (both translation and rotation), accompanied by cooperative relaxation ('local melting') of several surfactant chains. An estimate of the time is obtained by using an effective Rouse chain model of chain dynamics, coupled with a mean first passage time calculation. The second explanation invokes self-diffusion of the (di)polar probe itself from a less polar to a more polar region. This may also involve cooperative motion of the surfactant chains in the hydrophobic core, if the probe has a sizeable distribution inside the core prior to excitation, or escape of the probe to the bulk from the surface of the self-assembly. The second mechanism should result in the narrowing of the full width of the emission spectrum with time, which has indeed been observed in recent experiments. It is argued that both the mechanisms may give rise to an ultraslow time constant and may be applicable to different experimental situations. The effectiveness of solvation as a dynamical probe in such complex systems has been discussed.
\end{abstract}

Keywords. Ultraslow solvation dynamics; heterogeneous environments; polar probe.

\section{Introduction}

Time-dependent Stokes shift of fluorescence from a probe dye molecule has provided a wealth of information on the dynamics of dipolar liquids. ${ }^{1-11}$ In these experiments, a sudden (instantaneous) change in the charge distribution of the probe molecule is created optically using an ultrashort laser pulse. Subsequent change in the energy of the probe solute is monitored through the fluorescence frequency of the probe. Solvation time correlation function is defined as $^{1-3}$

$$
S(t)=(v(t)-v(\infty)) /(v(0)-v(\infty)),
$$

where $v(t)$ is the average frequency at a time $t$ of the fluorescence spectrum from the probe molecule. $S(t)$ evolves from unity at time $t=0$ to zero as time goes to infinity. The time dependence of $S(t)$ provides detailed information of the time-dependent response of the liquid to the sudden change in the charge distri-

*For correspondence bution in the probe. Since energy derives contributions from interactions with many solvent molecules, this response is collective. If the perturbation is small, the dynamics described by $S(t)$ is expected to be equivalent to the decay of the equilibrium time correlation function, $C(t)$, which is given by

$$
C(t)=\left\langle\delta E_{\text {solv }}(0) \delta E_{\text {solv }}(t)\right\rangle /\left\langle\delta E_{\text {solv }}(0) \delta E_{\text {solv }}(0)\right\rangle,
$$

where $\delta E_{\text {solv }}(t)$ is the fluctuation in the solvation energy about the equilibrium solvation energy. Detailed knowledge of the time-dependence of $C(t)$ is of fundamental importance to understand the solvent effects in many chemical processes, such as electron transfer, dephasing.

The microscopic processes involved in the solvation of a polar solute can be quite different in a heterogeneous environment. This is because in a heterogeneous environment, solvation energy of a polar probe derives contribution from several different sources. Thus, the solvation energy may be decomposed as

$$
\Delta E(t)=\sum_{i} \Delta E_{i}(t),
$$


where $\Delta E_{i}$ denotes contribution of the $i$ th component. The different contributions include interaction of the polar probe with the surface charges (e.g., polar head groups), the interfacial and the bulk water and the counterions. The relative amplitude of the contribution may depend on the location of the probe. An organized assembly is heterogeneous on a length scale longer than a molecule. As a result, an almost universal feature of solvation dynamics in a organized assembly is the highly non-exponential nature of the solvation energy relaxation. Thus, time-dependent response of such a system may be represented as

$$
C(t)=\sum_{i} a_{i} \exp \left(-t / \tau_{i}\right),
$$

where $\tau_{i}$ is the time constant of the $i$ th contribution.

Recently, there have been a large number of studies on solvation dynamics in various media, ranging from simple liquids (such as water, acetonitrile) to complex systems (micelles, vesicles, proteins). Among simple liquids, solvation dynamics studies in liquid water have attracted special attention for the ubiquitous role of water in biology. ${ }^{4-9}$ Solvation in pure water shows ultrafast solvation, with the initial component in the few tens of femtoseconds. In bulk water, even the slowest component is only about $1 \mathrm{ps.}^{4-9}$ However, in a restricted environment, the long time part of solvation dynamics slows down dramatically. ${ }^{8-23}$ At the surfaces of proteins, solvation dynamics displays a component which is slower by one to two orders of magnitude with time constants ranging from few tens to few hundreds of picoseconds. ${ }^{11-15}$ The ultraslow component in a cyclodextrin cavity is nearly 1000 times slower than that in bulk water. ${ }^{16}$ This dramatic slowing down of solvation dynamics is attributed to the freezing of translational (and rotational) motions of water molecules inside the cyclodextrin cavity. ${ }^{17}$ However, in the case of solvation dynamics in DNA, ${ }^{18}$ aqueous polymer solutions, ${ }^{19}$ micelles, ${ }^{20-24}$ reverse micelles ${ }^{25-28}$ and some more recent studies of protein solvation dynamics, a definite ultraslow component even greater than $1 \mathrm{~ns}(1000 \mathrm{ps})$ has been observed.

There is yet no satisfactory explanation of the origin of this ultraslow component. The existence of such a slow component is indeed surprising. We have earlier proposed a model involving dynamic equilibrium between free and bound water molecules in the vicinity of a biological macromolecule. ${ }^{12}$ According to this model the slow component of relaxa- tion depends on the binding energy, i.e. free energy difference $\left(\Delta G_{b f}^{0}\right)$ between bound and free water. In the limit of high binding energy $\left(k_{f b}>>k_{b f}\right)$ the time constant for the slowest component is given by

$$
k_{\text {slow }} \approx k_{b f}^{-1},
$$

where $k_{b f}$ denotes bound-to-free interconversion.

According to this model, the activation energy for bound-to-free interconversion is $\Delta G_{b f}^{0}+\Delta G^{\ddagger}$ where $\Delta G^{ \pm}$is the activation energy for free-bound interconversion. ${ }^{12}$ The dynamic exchange model is vindicated by a recent computer simulation ${ }^{22}$ and experimental determination of activation energy in solvation dynamics. ${ }^{23}$ According to the computer simulation the hydrogen bond energy of the interfacial water molecules and the polar head groups is stronger by $7-8 \mathrm{kcal} \mathrm{mol}^{-1}$ compared to bulk water. ${ }^{23}$ From temperature variation of solvation dynamics in a triton X-100 (TX) micelle has been found to involve an activation energy of $9 \mathrm{kcal} \mathrm{mol}^{-1}$ and a positive entropy of activation. ${ }^{23}$ However, although, the dynamic exchange model is adequate to explain the slow component in 30-500 ps time scale, it cannot satisfactorily explain the ultraslow component. The reason may be that this model completely ignores the role of motion of the macromolecular chains and diffusion of the probe itself in a heterogeneous environment.

It may be noted that the translational diffusion of the water molecules in many organized assemblies has been studied MD simulations. ${ }^{29-32}$ According to these simulations, the translational diffusion coefficients of water in the immediate neighbourhood of a protein, ${ }^{29,30}$ micelle, ${ }^{22,23}$ and lipid membrane ${ }^{31,32}$ and is 2-6 times slower than that in the bulk water. However, the above estimate is only an average which is dominated by fast-moving molecules and does not provide a correct picture of the slow dynamics of water at the surface. Translational diffusion deep inside the hydration layer of such a system may be many times slower.

In this work, we consider the role of chain dynamics and self-diffusion of probe in explaining the ultraslow component of solvation dynamics in an organized assembly.

\section{Theoretical formulation}

Before we discuss the theoretical models we should emphasize that an organized assembly is heterogeneous on a mesoscopic length scale, that is in the 
scale of 30-100 $\AA$. The equilibrium solvation energy of the charged probe is different in different phases because the polarity and the effective dielectric constant of the individual phases are different. For example, the solvation energy of a polar probe inside the hydrocarbon core of a micelle is expected to be much less than that at the micelle-water interface while the latter is again smaller than that in the bulk water. For simplicity, we consider a two phase system, with water being the outer phase. We also assume that the probe is hydrophobic in its ground state. Therefore, it is distributed primarily in the interface and also inside the hydrophobic core. The excited state of the probe, however, is polar (dipolar or charged).

The structure of the hydration shell of many other macromolecules have been studied by computer simulations ${ }^{22,24,27,31}$ and neutron scattering. ${ }^{35}$ The simulations indicate that in the hydration layer the macromolecules or surfactants are connected by hydrogen bond bridges involving water molecules (water bridges). The exact number of water molecules and water bridges vary from one surfactant to another. A single water molecule may form an intramolecular hydrogen bond bridge between two oxygen atoms of the same surfactant chain or an intermolecular hydrogen bond bridge between two neighbouring surfactant molecules. Tasaki ${ }^{33}$ identified two kinds of water bridges-one between two oxygen atoms of the same chain and another between an oxygen atom of the chain and the oxygen atom of another water molecule. Bandyopadhyay et $a l^{34}$ reported one hydrogen atom forms a hydrogen bond bridge between the two oxygen atoms of the same chain of a surfactant. In the case of a lipid (DMPC) bilayer about $70 \%$ of the surfactant (DMPC) molecules are found to be linked by either single or multiple intermolecular water bridges. ${ }^{31}$ In many cases, two DMPC molecules are simultaneously linked by 2,3 and in rare cases by 4 parallel water bridges. ${ }^{31}$ Such double or multiple hydrogen bonding gives certain amount of stability to these water molecules. Also the clustering of the water molecules around a macromolecule leads to close packing and a density higher ( 1.25 times $)$ than that in bulk water. ${ }^{29}$

\subsection{Model 1: Dynamics of surfactant chains with buried, slow water}

In this section, we consider the response of the buried water following creation of the dipole in terms of cooperative chain melting. Subsequent to the creation of the polarization field by the external probe near the surface, the buried water molecules inside a micelle (or in general in an organized assembly) would need to reorient to minimize their energy. However, reorientation would require breaking of hydrogen bond of a water molecule with another water molecule or with the surfactant and reformation of hydrogen bond with the dipole created (figure 1). This involves not only breaking and formation of hydrogen bonds but also rotation and migration of water molecules inside the layer and also of 'breathing' motion of the surfactant chains (figure 1). Dynamics of such a processes is expected to be rather slow and should be related to the dynamics of the surfactant chain.

In order to estimate the relaxation of the buried water molecules we assume the following simple model. We assume that the water molecules need to undergo a reaction between two minima which involves an activation barrier of $\sim 9 \mathrm{kcal} / \mathrm{mole}$ and a positive entropy factor. ${ }^{23}$ This activation energy is not fully known but can be guessed with reasonable accuracy. Next, we assume that the breakage of the hydrogen bond and its realignment or relocation requires cooperative motion of the nearest neighbour surfactant chains, may be two in number or greater. Thus, what we propose is a local melting in the hydrophobic core (figure 1). In the following we present a quantitative estimate of the time required for such melting. Note that this will be a slow process and is expected to be the rate limiting step in the solvation energy relaxation in the hydration shell.

In order for a cooperative melting to occur, one requires phase coherence in the motion of all the neighbouring chains. We model this by assuming that each monomer of the chain has locally $z$ number of states (chain conformations). Next, one needs to estimate the size of the region that can melt cooperatively. Let us assume that this number be denoted by $N$. If $\tau_{0}$ is the time required for transition from the ground to the excited state, then the time required for the phase coherence required for local melting is

$$
\tau=z^{N} \tau_{0}
$$

For convenience we shall assume that $z=2$, with a ground state denoted by ' 0 ' and the excited state by ' 1 '. If we assume that the fluorescent solvation probe is surrounded by two surfactant chains, then the lowest size that can melt is a cell which contains 6 monomers, that is $N=2$. Note that this is the lowest 


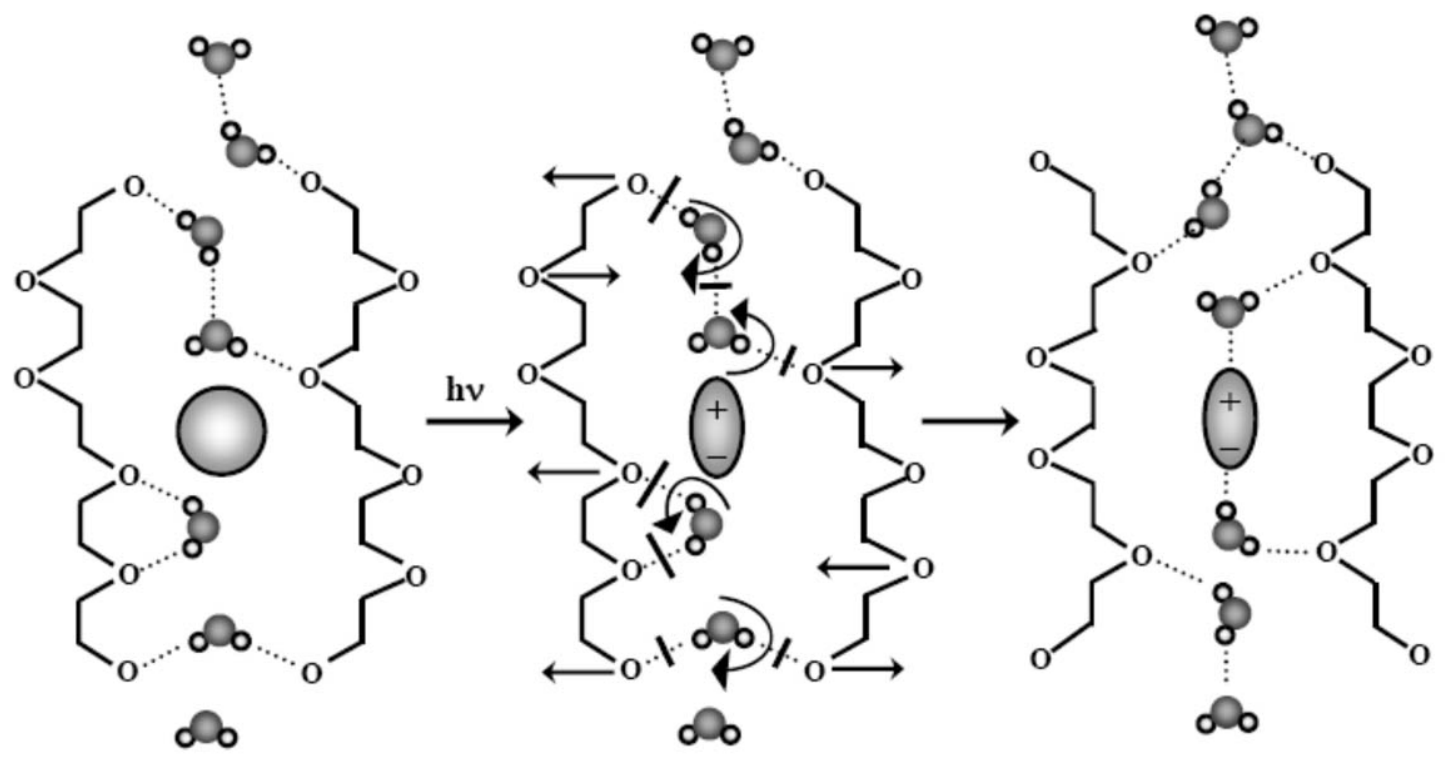

Figure 1. A schematic illustration of the role of buried water in the solvation dynamics of a probe which is initially located inside the self-assembly. This particular example illustrates the possible rearrangement of water molecules in a micelle of surfactant of polyethylene glycol (polyoxyethylene surfactant), ${ }^{34}$ subsequent to optical excitation.

size and this can only be larger because of the involvement of more than 2 chains. It is difficult to obtain an estimate of $\tau_{0}$ from any microscopic considerations because of the complexity of interactions and the highly condensed state of the hydration layer. We thus take recourse to the Rouse chain dynamics ${ }^{10}$ and assume that it can provide the estimate via the largest wave number fluctuation relaxation time.

$$
\tau_{0}=1 / \lambda_{N-1}=\left(3 D_{0}\right)^{-1}[N b /(N-1) \pi]^{2} .
$$

For TX-100, $N \approx 10$. We next need an estimate of $D_{0}$ which we obtain by using Stokes-Einstein relation $\left(D_{0}=k T / 6 \pi \eta_{\text {eff }} r\right)$ with effective viscosity of the hydration layer, $\eta_{\text {eff }}$ and radius $r$ of the monomer $\left(\mathrm{C}_{2} \mathrm{H}_{4} \mathrm{O}\right.$, in the case of the triton $\mathrm{X}-100$ micelle) unit.

The logic of the above analysis is that breaking of the hydrogen bond requires local excitation of the nearby surfactants. This excitation we calculate by using the normal modes of the surfactant chains and a first passage time calculation. We assume that these normal modes remain unperturbed by the presence of the others.

The advantage of the above simple expressions is that we can now easily estimate the relaxation time. If we assume that the radius of the monomer for a TX micelle $\left(\mathrm{C}_{2} \mathrm{H}_{4} \mathrm{O}\right)$ unit is $3 \AA$ and the effective viscosity $0.3 \mathrm{cP},{ }^{36}$ then the above expression gives a value of $\tau_{0} \approx 0.5 \mathrm{~ns}$. Use of this time constant in (6) gives a value of about $2 \mathrm{~ns}$ for the formation of the local excited state. So, this is the approximate theoretical estimate of the ultraslow component arising from the co-operative chain melting in the hydration layer. Note that the time required for breaking of the hydrogen bond is much smaller. It is about 10 $100 \mathrm{ps}$ for a single hydrogen bond and somewhat longer for the double bond which needs to be broken consecutively and which can be further slowed down by reformation.

The above estimate is certainly approximate and depends on the parameters chosen. Still we believe that we have obtained a reasonable number for the rate constant of this complex process of cooperative melting. The main features of this mechanism can be summarized as the lengthening of time of relaxation of deeply buried water in an organized assembly (such as a triton $X-100$ micelles) due to high degree of cooperatively required for these water molecules to participate in the solvation dynamics.

\subsection{Model 2: Self-diffusion of the probe}

The second explanation invokes the motion of the probe itself. Inside an organized assembly the local polarity or the dielectric constant of confined water 
molecules varies strongly over distance from the polar head groups. The large dielectric constant of water arises largely from the self-polarization of water molecules by neighbouring water molecules. ${ }^{37}$ In an organized assembly, the hydrogen-bond network of water is disrupted and water molecules become bound to the macromolecule by hydrogen bond. As a result, the self-polarization of water molecules is prevented. In principle, this effect is minimized at large distances from the surfactant and, hence, the local dielectric constant increases with distance from the surfactant chain. The variation of the local dielectric constant in an inhomogeneous medium such as a vesicle gives rise to the rededge excitation shift (REES). ${ }^{38,39}$

An organized assembly usually consists of a large hydrophobic core, and thus a fluorescent probe used for solvation dynamics studies is largely hydrophobic in its ground state. In the model involving selfdiffusion we assume that in the ground state a significant percentage of the probe molecules are trapped inside the relatively less polar region of the micelles/reverse micelles with a major portion of the probe inside the hydrocarbon chains. Subsequent to the excitation, the dipole moment of the probe increases substantially. Then the probe (which is initially in a less polar region) diffuses to a more polar region.

Experimentally, self-diffusion manifests in narrowing of the emission spectrum with increase in time (full width at half maxima, $\Gamma$ ). Recently, several

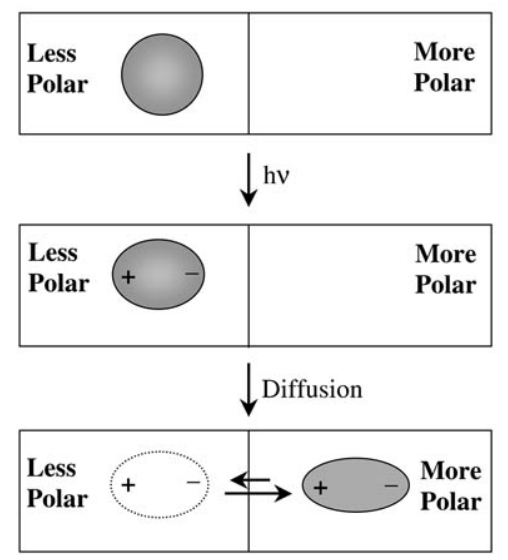

Figure 2. A schematic illustration of probe motion subsequent to optical excitation of the probe to a more polar state. A polar probe undergoes diffusion from a less polar to a more polar region in an organized assembly. The time dependence of the subsequent increase in the solvation stabilization energy is thus governed by probe diffusion in the confined system. groups have reported decay of $\Gamma$ with time $(t) .{ }^{28,40}$ Qualitatively, this may explained as follows. At $t=0$, the probe molecules in different environments are excited simultaneously. Due to the superposition of the emission spectra in different environments at short times, the spectral width of the emission spectrum is very large. Following electronic excitation the probe molecule becomes highly polar and hence, after excitation the probe molecules from all locations move towards the most polar region (figure 2). After a sufficiently long time, all the probe molecules reach the most polar region and all of them experience a more or less uniform environment. This results in a small $\Gamma$. Thus the time constant of decay of $\Gamma$ may be ascribed to self-diffusion of the probe ${ }^{28,40}$ Hof et $a l^{40}$ reported that in lipid vesicles, for some probes (9-AS, $\mathrm{C}_{17} \mathrm{DiFu}$, laurdan) the change in $\Gamma$ is negligible while for some other probes (e.g. prodan) there is an overall decrease by $20-25 \%$. They, however, did not extract the time constant of the decay of $\Gamma(t)$ and did not discuss the physical origin of this large decrease in $\Gamma$ with time. In figure 3 the decay reported by Hof et $a l^{40}$ is fitted to bi-exponential with time constants 155 ps $(20 \%)$ and $1850 \mathrm{ps}(80 \%)$, thus showing a time constant on the nanosecond time scale. Dutta et $a l^{28}$ showed that in a reverse micelle, $\Gamma(t)$ of DCM exhibits a decrease by as much as $70 \%$. They also showed that the time constant of decay of $\Gamma(t)$ of DCM is similar to that of $C(t)$ and hence, in this case the slow solva

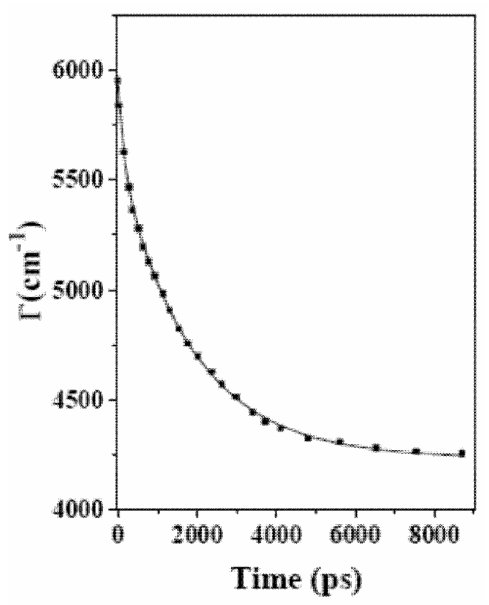

Figure 3. The time dependence of the full width at half maxima, $\Gamma$, of the emission spectrum of prodan in a lipid vesicle. The experimental results of Hof et $a l^{40}$ have been fitted to a bi-exponential form with time constants $155 \mathrm{ps}$ (20\%) and 1850 ps (80\%), thus showing a time constant in nanosecond time scale. The time dependence of $\Gamma(t)$ is due to the diffusion of the probe. 
tion dynamics is attributed to self-diffusion of the probe (DCM). ${ }^{28}$

It should be noted that a time-dependent change in spectral width is observed only in selected cases. For instance, the same probe (DCM) which exhibits time-dependent change in $\Gamma$ in a reverse micelle does not show similar behaviour when entrapped in a bile salt micelle. ${ }^{28}$ It is argued that in the latter case the probe is confined in a very long $(5 \mathrm{~nm})$ cylindrical water-filled core. As a result, within its excited state lifetime, the probe does not come out in bulk water and does not experience a large variation of local polarity.

Note, that most dyes are distributed over both polar and non-polar regions. If in the ground state a dye is localized in the polar region, after excitation it does not undergo self-diffusion and does not show a nanosecond component. Only those dyes which are initially in the non-polar region, migrate to a polar region after excitation and hence, display an ultraslow component.

Several groups have analysed fluorescence anisotropy decays in an organized assembly in terms of the 'wobbling-in-cone' model to estimate the diffusion coefficient $\left(D_{\|}\right)$for translation parallel to the surface of an organized assemble. ${ }^{41}$ It is observed that $D_{\|}$is smaller than that in bulk water $\left(\sim 10^{-5} \mathrm{~cm}^{2} \mathrm{~s}^{-1}\right)$ only by a factor of $2-5 .^{41}$ Thus the probe undergoes diffusion over a distance $\left.\left(<z^{2}\right\rangle=2 D_{\|} t\right)$ of about $10 \AA$ in one ns. The diffusion from non-polar to polar region may be in a direction normal to the surface of a micelle (or other organized assembly) and the corresponding diffusion coefficient $D_{\perp}$ may not be equal to $D_{\|}$.

We now present a molecular theory for the above dynamical process, namely, self-diffusion in an inhomogeneous medium A starting point for developing such a molecular theory is to use a time-dependent density functional $(\rho)$ theory which gives the following expression for the position $(X)$ dependent energy of interaction of a probe with the surrounding medium

$$
\begin{aligned}
E(X, t) & =\int \mathrm{d} X^{\prime} C_{P S_{i}}\left(X-X^{\prime}\right) \delta \rho_{S_{i}}\left(X^{\prime}, t\right) \\
& -\int \mathrm{d} X^{\prime} C_{P w}\left(X-X^{\prime}\right) \delta \rho_{w}\left(X^{\prime}, t\right),
\end{aligned}
$$

where $C_{P S i}$ is the two particle direct correlation function between the polar probe and the $i$ th group of the surfactant/polymer and $C_{P w}$ is the probe-water direct correlation function. In the inhomogeneous system, these pair correlation functions themselves depend on the location of the probe because density of surfactant or water is probe dependent. This makes a quantitative evaluation of detailed dynamics a prohibitively difficult task. However, we can make some progress because of the separation of time scales among the processes that are involved. Since the diffusion of the probe is a slow process, we may approximate the solvation time correlation function by

$$
\langle E(0) E(t)\rangle=\int \mathrm{d} x P(x, t)\langle E(x, 0) E(E(X, t)\rangle,
$$

where $P(X, t)$ gives the time evolution of the probe's location. A semi-quantitative description of the dynamics of the above process can be achieved by using a Smoluchowski level description where the probability distribution of the solute probe may follow the following equation of motion,

$$
\frac{\partial P}{\partial t}=D \frac{\partial^{2}}{\partial X^{2}} P-\frac{D}{k_{B} T} \frac{\partial}{\partial X}(F P),
$$

where the force $F$ is due to the potential gradient felt by the solute dipole created by the optical excitation. This equation gives the time scale of the evolution of the probability distribution under the force field. The initial and the final distributions are obviously different and are given by,

$$
\begin{aligned}
& P_{0}(X, t)=n_{0} \exp \left(-\beta V_{0}(X)\right), \\
& P_{E q}(X)=n_{0} \exp \left(-\beta V_{E q}(X)\right) .
\end{aligned}
$$

With $(\beta=1 / k T)$, the initial and final potentials can in principle be calculated from the density functional theory outlined above but that is still rather hard. A simple way to express the potential $V$ is to invoke a continuum model with a space dependent dielectric constant $\varepsilon(X)$ (see figure 2). The potential energy at a position $X$ of an ionic probe can then be crudely approximated by

$$
V(X)=-q^{2} / a[1-1 / \varepsilon(X)] .
$$

The solvation dynamics probed is then determined by the diffusion of the probe from the lower to the larger dielectric constant. Inside the hydrophobic core, the dielectric constant can be small, about 3-5, while in the bulk, it is close to 80 . The radius $a$ is 
about 3-5 A. The Smoluchowski equation can now be solved to obtain the following expression

$$
\tau_{s}\left(X_{0}, b\right)=\frac{1}{D}\left[\int_{X_{0}}^{X_{M}} \mathrm{~d} y \exp (\beta V(y)) \int_{b}^{y} \mathrm{~d} z \exp (-\beta V(z)] .\right.
$$

where $\beta=1 / k_{B} T$. $X_{0}$ is the starting position, $X_{M}$ is the final position and $b$ is a position left of $X_{0}$ where probability is zero. Therefore, the average time is integration over the initial probability distribution, $P_{0}(X)$. The position $b$ could be taken as at $4-6 \AA$ inside the initial position of the probe inside the micelle. $D$ is an average diffusion constant which should in principle also have an $X$ dependence which is neglected here for simplicity. Typically viscosity in the core is expected to be about twice that of liquid water.

If we assume that the probe is a sphere of radius $a=3 \AA$, the partial charge created on the probe at $t=0$ is $0.5 \mathrm{esu}$, the probe diffuses as distance of $4 a$ with an average diffusion coefficient of $10^{-5} \mathrm{~cm}^{2} / \mathrm{s}$, then we obtain an estimate of $740 \mathrm{ps}$ from the above expressions. This is of course a crude estimate of the time scale involved, meant to demonstrate that the proposed mechanism can indeed give time constants of the order observed in many experiments. The main idea is that subsequent to excitation, the probe may undergo a forced (or, biased) diffusion towards more polar region, as illustrated in figure 2 .

\section{Concluding remarks}

The above analysis suggests that in some cases, there could be more than one mechanism that can give rise to an ultraslow component observed in recent experiments and the relative contributions can be hard to estimate. One could, however, roughly estimate the time scales responsible for the different slow mechanisms. It is clear that the dynamic exchange between the free and the bound states of water molecules at the surface is important at relatively short times. We expect this mechanism to be important up to about 500 ps or so and this model should dominate in the 50-500 ps range. The case of TX100 is however, is extraordinary because here the water molecules inside the hydration shell are highly restricted and the dynamic exchange is expected to be on the slow side. The self-diffusion of probe should contribute next in order of time scale. As dis- cussed, this should be in the 1-100 ns range, depending on the width of the hydrophobic core that the probe needs to diffuse through. The role of the selfdiffusion of the probe could be estimated by the variation of the width $\Gamma$ at half-maximum of TRES. ${ }^{30,31}$ This could give an estimate of the relative importance of probe self-diffusion in the slow solvation.

There could be a third mechanism for ultraslow solvation which involves the motion of the protein or the self-assembly. At surfaces of proteins, the solvation of a probe may derive contribution from the motion of the protein side chains. Dielectric relaxation times in an aqueous solution a protein span a wide range. The different components of dielectric relaxation may classified as: (1) reorientation of bulk water (8 ps); (2) overall tumbling or reorientation of the entire protein molecule (10-100 ns) and (3) relaxation of water associated with the protein (10-150 ps). ${ }^{9,12,42,43}$ According to the Stokes-Einstein relation, the overall tumbling time $\left(\tau_{m}\right)$ of a biological system of volume $V$ is given by,

$$
\tau_{m}=\eta V / k_{B} T
$$

where $\eta$ is the viscosity of water. From this relation, $\tau_{m}$ may be calculated if the volume of the macromolecule is known. For instance, the protein human serum albumin (HSA) is an ellipsoidal of dimension $8 \times 8 \times 3 \mathrm{~nm}$. Thus for HSA $\tau_{m}$ is $25 \mathrm{~ns}$ in bulk water. It should be emphasized that if the probe is attached to the protein surface, the overall tumbling gives rise to a relative motion of the probe and the water molecules surrounding the protein.

Using the Stokes-Einstein relation, Wand et $a l^{45}$ calculated that for a protein of mass $50 \mathrm{kDa}$ and radius $26 \mathrm{~A}$ the overall tumbling time is $15 \mathrm{~ns}$ in water, and that in the case of a reverse micelle, the tumbling time (estimated for NMR relaxation) decreases with viscosity of bulk hydrocarbon. The dielectric relaxation of aqueous proteins exhibits a $10-100 \mathrm{~ns}$ component arising from tumbling of proteins. ${ }^{9}$ Thus one possible source of the very long component (e.g. $10 \mathrm{~ns}$ reported for HSA) could be tumbling of the protein.

One way to check the role of overall tumbling is to create a situation when overall tumbling of the protein is impossible. If a protein is confined inside a water pool of the reverse micelle or a lipid, the lipid vesicle or the reverse micelle tumbles as a whole. The radius $\left(r_{h}\right)$ of a DMPC vesicle is about $15 \mathrm{~nm} .{ }^{44}$ Thus, the volume of the vesicle is about 
140 times than that of a single HSA molecule. From (9), $\tau_{m}$ is $3500 \mathrm{~ns}$ for the lipid vesicle. Evidently, overall tumbling of the lipid vesicle $\left(\tau_{m}=3500 \mathrm{~ns}\right)$ is impossible within the excited lifetime (1-2 ns) of the fluorescent probe (DCM).

All the mechanisms discussed above provide explanations for the observed ultraslow time constant in the solvation dynamics of an external probe in self-assemblies. This also highlights the difficulty of using solvation dynamics as a sensitive method to study dynamics in such systems. One may need to combine it with various other experimental techniques (such as REES, dielectric relaxation, NMR) and also with computer simulations and theoretical studies to gain an understanding of the relative role of a specific mechanism in a given situation. However, slow dynamics can offer valuable insight into the dynamics of the self-assembly itself.

\section{Acknowledgement}

This work was supported in part by grants from the Department of Science and Technology and the Council of Scientific and Industrial Research, India.

\section{References}

1. Maroncelli M, McInnis J and Fleming G R 1989 Science 2431674

2. Maroncelli M 1993 J. Mol. Liq. 571

3. Bagchi B 1989 Annu. Rev. Phys. Chem. 40115

4. Fecko C J, Eaves J D, Loparo J J, Tokmakoff A and Geissler P L 2003 Science 3011698

5. Jimenez R, Fleming G R, Kumar P V and Maroncelli M 1994 Nature (London) 369471

6. Nandi N, Roy S and Bagchi B 1995 J. Chem. Phys. 1051390

7. Jarzeba W, Walker G C, Johnson A E, Kahlow M A and Barbara P F 1988 J. Phys. Chem. 927039

8. Bagchi B 2003 Annu. Rep. Prog. Chem. Sect. C99 127

9. Nandi N, Bhattacharyya K and Bagchi B 2000 Chem. Rev. 1002013

10. Pal S K, Peon J, Bagchi B and Zewail A H $2002 J$. Phys. Chem. B106 12376

11. Otting G, Liepinsh W and Wuthrich K 1991 Science 254974

12. (a) Nandi N and Bagchi B 1997 J. Phys. Chem. B101 10954; (b) Nandi N and Bagchi B 1998 J. Phys. Chem. A102 8217

13. Jordandies $X$ J, Lang $M J$, Song $X$ and Fleming G R 1999 J. Phys. Chem. B103 7995

14. Pal S K and Zewail A H 2004 Chem. Rev. 1042099
15. Mandal D, Sen S, Sukul D, Bhattacharyya K, Mandal A K, Banerjee R and Roy S 2002 J. Phys. Chem. B106 10741

16. Vajda S, Jimenez R, Rosenthal S J, Fidler V, Fleming G R and Castner E W Jr 1995 J. Chem. Soc. Faraday Trans. 91867

17. Nandi N and Bagchi B 1996 J. Phys. Chem. 100 13914

18. (a) Gearheart L A, Somoza M M, Rivers W E, Murphy C J, Coleman R S and Berg M A $2003 \mathrm{~J}$. Am. Chem. Soc. 125 11812; (b) Brauns E B, Madaras M L, Coleman R S and Berg M A 2002 Phys. Rev. Lett. 88 158101-1-4

19. Frauchiger L, Shirota H, Uhrich K E and Castner E W Jr 2002 J. Phys. Chem. B106 7463

20. Hara K, Kuwabara H and Kajimoto O 2001 J. Phys. Chem. A105 7174

21. Mandal D, Sen S, Tahara T and Bhattacharyya K 2002 Chem. Phys. Lett. 35977

22. (a) Balasubramanian S, Pal S and Bagchi B $2003 \mathrm{~J}$. Phys. Chem. B107 5194; (b) Balasubramanian S, Pal S and Bagchi B 2002 Phys. Rev. Lett. 89 115505-1-4

23. Sen P, Mukherjee S, Halder A and Bhattacharyya K 2004 Chem. Phys. Lett. 385357

24. Bruce C D, Senapati S, Berkowitz M L, Perera L and Forbes M D E 2002 J. Phys. Chem. B106 10902

25. Lundgren J S, Heitz M P and Bright F V 1995 Anal. Chem. 673775

26. Corbeil E M, Riter R E and Levinger N E $2004 \mathrm{~J}$. Phys. Chem. B108 10777

27. Faeder J and Ladanyi B M 2002 J. Phys. Chem. B104 1033

28. Dutta P, Sen P, Mukherjee S, Halder A and Bhattacharyya K 2003 J. Phys. Chem. B107 10815

29. Levitt M and Sharon R 1988 Proc. Natl. Acad. Sci. USA 857557

30. Bellisent-Funel M-C 2000 J. Mol. Liq. 8439

31. (a) Pasenkiewicz-Gierula M, Takaoka V, Miyagawa, H, Kitamura K and Kusumi A 1997 J. Phys. Chem. A101 3677; (b) Rog T, Murzyn K and PasenkiewiczGiurela M 2002 Chem. Phys. Lett. 352323

32. Konig S, Sackmann E, Richter D, Zorn R, Carlile C and Bayerl T M J 1994 Chem. Phys. 1003307

33. Tasaki K 1996 J. Am Chem. Soc. 1188459

34. (a) Bandyopadhyay S, Tarek M, Lynch M L and Klein M L 2000 Langmuir 16 942; (b) Allen R, Bandyopadhyay S and Klein M L 2000 Langmuir 16 10547; (c) Bandyopadhyay S and Chanda J 2003 Langmuir 1910443

35. Berr S S, Caponetti E, Jones R R M, Johnson J S and Magid L J 1987 J. Phys. Chem. 915766

36. Pal S K, Datta A, Mandal D and Bhattacharyya K 1998 Chem. Phys. Lett. 288793

37. Rick S W, Stuart S J and Berne B J $1994 \mathrm{~J}$. Chem. Phys. 1016141

38. (a) Chattopadhyay A and Mukherjee S 1999 J. Phys. Chem. B103 8180; (b) Chattopadhyay A, Mukherjee $\mathrm{S}$ and Raghuraman H $2002 \mathrm{~J}$. Phys. Chem. B106 13002; (c) Demochenko A P and Ladokhin A S 1988 Eur. Biophys. J. 15569 
39. (a) Satoh $\mathrm{T}$, Okuno $\mathrm{H}$, Tominaga $\mathrm{K}$ and Bhattacharyya K 2004 Chem. Lett. 33 1090; (b) Sen P, Satoh T, Tominaga K and Bhattacharyya K 2006 Chem. Asian J. 1188

40. Sykora J, Kapusta P, Fidler V and Hof M 2002 Langmuir 18571

41. (a) Quitevis E L, Marcus A H and Fayer M D $1993 \mathrm{~J}$. Phys. Chem. 97 5762; (b) Wittouck N W, Negri R M and De Schryver F C $1994 \mathrm{~J}$. Am. Chem. Soc. 116 10601; (c) Sen S, Sukul D, Dutta P and Bhattacharyya K 1994 J. Phys. Chem. A105 7495
42. Grant E H, Sheppard R J and South G P 1978 Dielectric behavior of biological molecules (Oxford: Clarendon)

43. Huscha T, Peytcheva A and Kaatze U 2002 J. Phys.Cond. Matter 149461

44. Bardos-Nagy I, Galántai R, Laberge M and Fidy J 2003 Langmuir 19146

45. Wand A J, Ehrhardt M R and Flynn P F 1998 Proc. Natl. Acad. Sci. USA 9515299

46. Dutta P, Sen P, Mukherjee S and Bhattacharyya K 2003 Chem. Phys. Lett. 382426 